\title{
EVIDÊNCIAS CIENTÍFICAS SOBRE A POLÍTICA NACIONAL DE ATENÇÃO A SAÚDE DA CRIANÇA
}

\author{
Paulo Arthur da Penha Lima*, Erika de Vasconcelos Barbalho** \\ Autor para correspondência: Erika de Vasconcelos Barbalho - Email: erikavascb@gmail.com \\ * Fisioterapeuta graduado pelas Faculdades INTA. Sobral - CE. Brasil \\ ** Fisioterapeuta. Residente em Saúde da Família. Professora do curso de Fisioterapia das Faculdades INTA. Sobral - CE. \\ Brasil
}

\begin{abstract}
Resumo
Como ferramenta do Sistema Único de Saúde, a Política de Atenção à Criança está relacionada à saúde materna, determinada como Política de Saúde Materno-Infantil. O cuidado à saúde da criança corresponde a um campo privilegiado dentro da atenção à saúde das populações. O objetivo do estudo foi descrever, por meio de uma revisão da literatura, um breve resgate das produções sobre a Política Nacional de Saúde da Criança no Brasil. Este estudo teve como característica a pesquisa bibliográfica, os descritores acessíveis na lista dos Descritores em Saúde (DeCS) da Biblioteca Virtual em Saúde (BVS), a saber: "criança", "saúde da criança", "sistema único de saúde" e seus correspondentes. Esses descritores foram úteis para busca nas bases de dados LILACS, Scielo, livros, revistas e cartilhas do Ministério da Saúde. Critérios de inclusão: artigos datados desde a implantação do Sistema Único de Saúde até a presente data; publicações em língua portuguesa; pesquisas que tivessem como foco principal as políticas direcionadas a saúde da criança e seu processo de construção histórica. Critério de exclusão: publicações que não estiveram em formato de artigo científico validados pela Capes, teses, dissertações, resenhas, editoriais e cartas. Pôde-se observar que os temas dos estudos encontrados tiveram como abordagem principal as políticas que englobam a assistência voltada para a saúde da criança, relatando os autores e anos de publicação, as intervenções adotadas dentro de cada programa sanitário e seus respectivos objetivos. Assim, este trabalho vem mostrar as evidências científicas a cerca da política de assistência infantil brasileira.
\end{abstract}

Palavras-chave: Criança; Saúde da criança; Sistema Único de Saúde. 


\title{
SCIENTIFIC EVIDENCE ON THE NATIONAL POLICY OF HEALTH WARNING CHILD
}

\begin{abstract}
As the Health System tool, the Child Care Policy is related to maternal health, determined to Maternal and Child Health Policy. The health care of the child corresponds to a privileged area within the health care of the population. The objective of the study was to describe, through a literature review, a brief redemption of productions on the National Health Policy of the Child in Brazil. This study characterized the literature, accessible descriptors in the list of Descriptors in Health (MeSH) of the Virtual Health Library (VHL), namely: "child", " children's health", "public health system" and its correspondents. These descriptors were useful for search in databases LILACS, SciELO, books, magazines and leaflets of the Ministry of Health Inclusion criteria: Articles dating from the implementation of the National Health System to date; publications in English ; research that had focused primarily on the policies directed to children's health and their historical construction process. Exclusion criteria: publications that have not been in scientific paper format validated by Capes, theses, dissertations, book reviews, editorials and letters. It was observed that the subjects of the studies found were mainly approach the policies that encompass focused assistance to the child's health, reporting the authors and years of publication, interventions adopted within each health program and their goals. This work goes to show the scientific evidence about the Brazilian child care policy.
\end{abstract}

Keywords: Child; Child health; Single system health.

\section{INTRODUÇÃO}

O Sistema de Saúde do Brasil (SUS) foi instituído pela Constituição Federal Brasileira de 1988, no artigo 198, em decorrência do movimento pela Reforma Sanitária e da luta pela redemocratização da nação. Foi, então, regulamentado com as leis n.8.142 e n.8.080, de 1990, tendo como princípios básicos: o atendimento integral, para vencer a separação entre os serviços preventivos e curativos; a descentralização, com propagação dos centros de poder entre as instâncias governamentais; e o controle social, descrito pelo trabalho popular no controle dos serviços de saúde. (1)

A partir daí, evidenciou-se a importância da promoção da saúde como direito essencial da população, cabendo ao Estado a compromisso de garan- ti-la universalmente. Sob a diretriz constitucional "saúde direito de todos e dever do Estado" preservou a assistência médico-sanitária integral, com acesso igual a todos os usuários aos serviços de saúde, sendo estes hierarquizados e a sua gestão descentralizada. Decretou-se que as ações de saúde poderiam estar submetidas a organismos do executivo com representação paritária entre usuários e os demais representantes do governo, em suas várias áreas, dos profissionais de saúde e dos prestadores de serviços públicos e privados. ${ }^{(2)}$

Uma das estratégias desenvolvidas pelo Ministério da Saúde (MS), a partir de 1984, pretendendo desenvolver a eficácia resolutiva dos serviços de saúde, foi intensificar os cuidados à criança e a mãe, 
elegendo cinco ações fundamentais de saúde que apresentaram bons resultados (promoção do aleitamento materno, imunizações, prevenção e controle das doenças de infecções respiratórias agudas e diarreicas e acompanhamento do crescimento e desenvolvimento). $\mathrm{E}$, diante disso, criou as normas técnicas, definiu instrumentos operacionais e promoveu a treinamento de recursos humanos para o desenvolvimento destas atividades. ${ }^{(3)}$

A primeira infância é vista atualmente como momento prioritário para intervenção, porque os pequenos indivíduos são vistos como flexíveis e suscetíveis à influência externa. $O$ indício de que o desenvolvimento cerebral é mais intenso nos primeiros 5 anos de vida, faz com que qualquer programa que vise ao desenvolvimento do cérebro seja avaliado de forma positiva, proporcionando uma melhora na educação e no cuidado da criança, de modo integral, principalmente para aquelas mais vulneráveis socialmente. ${ }^{(4)}$

O cuidado à saúde da criança corresponde a um campo privilegiado dentro da atenção à saúde das populações para que essa se desenvolva de forma fixa e eficaz, diante do conhecimento sobre as características ligadas à morbimortalidade, tais como aspectos biológicos, socioeconômicos e demográficos, é necessário destacar o papel que executam o sistema de saúde e os serviços. ${ }^{(5)}$

No SUS, a Política de Atenção à Criança está relacionada à saúde materna, determinada como Política de Saúde Materno-Infantil. A concepção mais difundida da criança como ser em desenvolvimento permanente resultou de um longo processo que incluiu modificações na organização da sociedade, desde o enfoque da esfera privada das famílias, obtendo as políticas públicas, que autorizam a adesão de práticas e normas para esses processos de transformação. Tais práticas contribuem para a prestação de uma saúde, assistência e educação, de caráter universal, exigido e sob a responsabilidade do Estado. ${ }^{(6)}$

Portanto, o objetivo do presente trabalho foi descrever, por meio de uma revisão da literatura, um breve resgate das produções sobre a Política Nacional de Saúde da Criança no Brasil.

\section{METODOLOGIA}

Este estudo tem como característica a pesquisa bibliográfica, pois procura explicar um problema a partir de referências teóricas publicadas em artigos, livros, dissertações e teses. Pode ser realizada independentemente ou a partir da pesquisa descritiva ou experimental. Em ambos os casos, busca-se conhecer e analisar as contribuições culturais ou científicas do passado sobre determinado assunto, tema ou problema. ${ }^{(7)}$

A estratégia de investigação baseado na revisão, busca manter os padrões de clareza, replicação e rigidez dos estudos primários. Desta maneira, para produzir a revisão, além de formular questões para orientação e hipóteses, é importante estabelecer critérios claros de exclusão e inclusão para constituir a amostra, definir as características dos estudos primários levantados, analisar dados agrupados, interpretar os resultados alcançados e, finalmente, expor a revisão. ${ }^{(8)}$

A questão que orientou a presente pesquisa foi: como se deu o processo histórico de implantação e implementação da atenção à saúde da criança no Brasil?

Para realizar a apuração na literatura foram escolhidos os descritores acessíveis na lista dos Descritores em Saúde (DeCS) da Biblioteca Virtual em Saúde (BVS), a saber: "criança", "saúde da criança", "sistema único de saúde" e seus correspondentes na língua portuguesa. Esses descritores foram úteis para a busca nas bases de dados bibliográficos: LILACS (Literatura Latino-americana e do Caribe), Scielo (Scientific Electronic Library Online), livros e, revistas e cartilhas do Ministério da Saúde. A coleta dos dados ocorreu no período de junho a setembro de 2014 .

Foram julgados os seguintes critérios que limitaram a busca inicial: a) artigos datados desde a implantação do Sistema Único de Saúde até a pre- 
sente data; b) publicações em língua portuguesa; c) pesquisas que tivessem como foco principal as políticas direcionadas a saúde da criança e seu processo de construção histórica.

Foram excluídas as publicações que não estiveram em formato de artigo científico validados pela Capes, teses, dissertações, resenhas, editoriais e cartas.

Depois do levantamento preliminar na base de dados selecionada, os resumos das publicações escolhidas foram revisados de modo a se poder aprimorar a escolha final dos dados que constituíram o corpo deste estudo.

Após aplicar os critérios de inclusão e exclusão e o refinamento posterior da pesquisa, os estudos foram catalogados conforme uma ficha que resume suas características gerais. Nessa ficha foram apontados: a) base de dados pesquisada; b) autores; c) dados referentes à publicação (ano e periódico); d) objetivo de cada política analisada.
Os resultados foram organizados de maneira a destacar as seguintes dimensões: a) apontar, de modo organizado, as publicações sobre a construção da Política Nacional de Saúde da Criança; b) analisar o conteúdo dos artigos quanto a seus objetivos, metodologia empregada e principais resultados encontrados; e, c) sintetizar os principais auxílios das publicações em relação ao tema estudado, buscando desenhar perspectivas de intervenção e futuras pesquisas.

\section{RESULTADOS E DISCUSSÃO}

No quadro abaixo (Quadro 1), pode-se observar que os temas dos estudos encontrados tiveram como abordagem principal os princípios que englobam a assistência voltada para a saúde da criança, relatando os autores e anos de publicação, a política abordada dentro do programa saúde da criança e seus respectivos objetivos.

Quadro 1 - Apresentação dos estudos selecionados de acordo com autor, ano de publicação, política assistencial e objetivos de cada estratégia política

(continua)

\begin{tabular}{|c|c|c|c|}
\hline AUTOR & ANO & POLITICA & OBJETIVO DE CADA POLITICA \\
\hline CARVALHAL, et.al. & 2013 & $\begin{array}{l}\text { Agenda de compromisso para } \\
\text { saúde integral da criança e } \\
\text { redução da mortalidade infantil }\end{array}$ & $\begin{array}{l}\text { A política tem como finalidade apoiar a } \\
\text { organização da assistência à população } \\
\text { infantil e possibilitar que os gestores } \\
\text { e profissionais de saúde identifiquem } \\
\text { as ações prioritárias para a saúde da } \\
\text { criança. }\end{array}$ \\
\hline $\begin{array}{l}\text { MENDES; SANTOS; } \\
\text { BRINGEL }\end{array}$ & 2013 & $\begin{array}{l}\text { Programa nacional de triagem } \\
\text { neonatal, triagem auditiva } \\
\text { neonatal e teste do reflexo } \\
\text { vermelho }\end{array}$ & $\begin{array}{l}\text { Detectar as doenças congênitas em fase } \\
\text { pré sintomática em todos os nascidos } \\
\text { vivos. }\end{array}$ \\
\hline CAVALCANTI, et. al. & 2013 & Rede Cegonha & $\begin{array}{l}\text { Ampliar a rede de assistência ao parto } \\
\text { e ao nascimento, garantindo vagas para } \\
\text { gestantes e recém-nascidos e evitar a } \\
\text { peregrinação da gestante por ocasião } \\
\text { do parto. }\end{array}$ \\
\hline GOULART, et. al. & 2008 & $\begin{array}{l}\text { Acompanhamento do } \\
\text { crescimento e desenvolvimento- } \\
\text { caderneta de saúde da criança e } \\
\text { do adolescente }\end{array}$ & $\begin{array}{l}\text { O acompanhamento e a vigilância do } \\
\text { crescimento e desenvolvimento infantil } \\
\text { para promover a saúde da criança }\end{array}$ \\
\hline
\end{tabular}


Quadro 1 - Apresentação dos estudos selecionados de acordo com autor, ano de publicação, política assistencial e objetivos de cada estratégia política

(conclusão)

\begin{tabular}{|c|c|c|c|}
\hline AUTOR & ANO & POLITICA & OBJETIVO DE CADA POLITICA \\
\hline TEMPORÃO & 2003 & $\begin{array}{l}\text { Programa nacional de } \\
\text { imunização }\end{array}$ & $\begin{array}{l}\text { Atingir metas de imunização para } \\
\text { redução de casos e erradicação de } \\
\text { doenças, afim de melhorar a qualidade } \\
\text { de vida da população }\end{array}$ \\
\hline $\begin{array}{l}\text { OLIVEIRA; } \\
\text { CAMACHO; SOUZA }\end{array}$ & 2005 & $\begin{array}{l}\text { Politica brasileira de promoção, } \\
\text { proteção e apoio ao aleitamento } \\
\text { materno. }\end{array}$ & $\begin{array}{l}\text { Melhorar o índice de amamentação, a } \\
\text { fim de criar um vincula afetivo com a } \\
\text { mãe, proteção e nutrição da criança. }\end{array}$ \\
\hline BRASIL & 2012 & $\begin{array}{l}\text { Promoção da alimentação } \\
\text { saudável }\end{array}$ & $\begin{array}{l}\text { Criar hábitos alimentares nos primeiros } \\
\text { anos de vida das crianças. }\end{array}$ \\
\hline FELISBERTO, et. al. & 2002 & $\begin{array}{l}\text { Atenção integrada as doenças } \\
\text { prevalentes na infância }\end{array}$ & $\begin{array}{l}\text { Redução da mortalidade infantil por } \\
\text { doenças mais comuns na infância } \\
\text { como diarreias, infecções respiratórias } \\
\text { agudas, desnutrição e malária. }\end{array}$ \\
\hline MENDES & 2012 & $\begin{array}{l}\text { Brasileirinhas e brasileirinhos } \\
\text { saudáveis: primeiros passos para } \\
\text { o desenvolvimento nacional }\end{array}$ & $\begin{array}{l}\text { Fortalecer o conjunto de esforço em } \\
\text { todo o pais para articulação, interação } \\
\text { e implantação de ações voltadas a } \\
\text { saúde da mulher e da criança até } 6 \\
\text { anos. }\end{array}$ \\
\hline SANTIAGO, et. al. & 2012 & Programa saúde na escola & $\begin{array}{l}\text { Contribuir para a formação integral de } \\
\text { estudantes de rede publica de educação } \\
\text { básica por meio de ações de prevenção, } \\
\text { de proteção e de atenção a saúde. }\end{array}$ \\
\hline BRASIL & 2010 & $\begin{array}{l}\text { Linha de cuidado para atenção } \\
\text { integral a saúde de crianças, } \\
\text { adolescentes e suas famílias em } \\
\text { situação de violência }\end{array}$ & $\begin{array}{l}\text { Visa garantia da continuidade do } \\
\text { atendimento e da articulação das } \\
\text { várias ações desenvolvidas pela rede } \\
\text { e cuidado em saúde com a proteção } \\
\text { social no território. }\end{array}$ \\
\hline RAPOSO & 2009 & \begin{tabular}{|l|} 
Política Nacional de Atenção \\
Integral a Saúde de Adolescentes \\
e Jovens
\end{tabular} & $\begin{array}{l}\text { Promover a atenção integral a saúde } \\
\text { de adolescentes e jovens de } 10 \text { a } 24 \\
\text { anos no âmbito da política nacional de } \\
\text { saúde, visando a promoção de saúde, a } \\
\text { prevenção de agravos e a redução da } \\
\text { morbimortalidade. }\end{array}$ \\
\hline
\end{tabular}

$\mathrm{Na}$ política de Agenda de Compromisso para Saúde Integral da Criança e Redução da Mortalidade Infantil, a assistência integral é o eixo condutor da agenda de compromisso. Trata-se da abordagem global da criança, contemplando todas as ações de saúde adequadas para promover respostas satisfatórias da produção do cuidado, visando a reorganização dos serviços de saúde nos três ní- veis de atenção por meio de linhas de cuidados que visam a integralidade. ${ }^{(9)}$

$\mathrm{Na}$ estratégia Linhas de Cuidado para a Atenção Integral a Saúde da Criança as estratégias fundamentais para a superação da desarticulação entre os diversos níveis de atenção em saúde, garantem a permanência da continuidade do cuidado inte- 
gral desde as ações de promoção até as de tratamento e reabilitação. ${ }^{(10)}$

Para melhor atender as necessidades da saúde neonatal e auxiliar na deteç̧ão precoce de doenças no primeiro mês de vida das crianças, os serviços de saúde se utilizam das ferramentas doutrinárias da Triagem Neonatal (teste do pezinho), Triagem Auditiva Neonatal (teste da orelhinha) e, mais recentemente, do Teste do Reflexo Vermelho Ocular (teste do olhinho), com o intuito de detectar o quanto antes alterações e diagnosticar doenças que podem comprometer o crescimento e desenvolvimento (CD) infantil. ${ }^{(11)}$

Recentemente o governo brasileiro propôs uma nova ação para a promoção da saúde das crianças, instituído, no âmbito do SUS, a Rede Cegonha, mediante a portaria $n^{\circ} 1459$, de 24 de junho de 2011. É uma rede de cuidados que visa assegurar à muIher o direito ao planejamento reprodutivo e a atenção humanizada a gravidez, ao parto e ao puerpério, bem como o direito ao nascimento seguro e ao crescimento e desenvolvimento saudável a criança de $O$ a 2 anos. ${ }^{(12)}$

A Rede Cegonha organiza-se a partir de quatro componentes: pré-natal, parto e nascimento, puerpério e atenção integrada à saúde da criança, sistema logístico- transporte sanitário e regulação, além de qualificar os profissionais de saúde ao atendimento à gestante a ao neonato. ${ }^{(13)}$

As políticas públicas voltadas a saúde da criança consideram o acompanhamento do crescimento e desenvolvimento infantil como eixo referencial para todo atendimento da criança em qualquer local que ela se apresente, seja em unidades de saúde, hospitais, escolas ou creches, pois crianças submetidas a adequadas condições de saúde crescem e se desenvolvem utilizando todo o seu potencial. ${ }^{(14)}$

$\mathrm{Na}$ adolescência, o acompanhamento do crescimento e desenvolvimento levam em consideração, além da estrutura e do peso as transformações corporais e a maturação dos órgãos sexuais provenientes da puberdade. É nessa fase que o organismo deixa de sofrer tantas influências externas com relação ao crescimento, sendo submetido a maior influência dos fatores orgânicos. ${ }^{(15)}$

Outra estratégia utilizada para reduzir as taxas de mortalidade infantil é a criação do Programa Nacional de Imunização (PNI) que é responsável por organizar, coordenar e operacionalizar a produção, o armazenamento e a distribuição de vacinas, bem como as ações de imunização em todo o território nacional, que visam estratégias fundamentais na promoção e proteção da saúde de crianças e adolescentes, com vista à prevenção de doenças e a redução de mortalidade infantil.(16)

O PNI determina a realização da cobertura vacinal de $100 \%$ das crianças brasileiras por meio do preenchimento do calendário vacinal da criança, que deve ser instituído em sua totalidade pelo sistema de saúde e pelas famílias. A cada período, esse calendário é analisado e modificado, com entrada e saída de vacinas de acordo com a situação epidemiológica das doenças imunopreveníveis no Brasil. (17)

A promoção ao aleitamento materno complementa os efeitos produzidos pelas vacinas, sendo um direito garantido por lei. Todos os profissionais da saúde devem defender, estimular, promover e apoiar a amamentação em todos os níveis de atenção a saúde. Considerando os benefícios que traz para a criança, a Organização Mundial da Saúde (OMS) e o MS recomendam que o aleitamento materno seja realizado de maneira exclusiva até os seis meses de vida e complementando com alimentação saudável até os dois anos. ${ }^{(18)}$

O direito a amamentação deve ser garantido por outros setores da sociedade, principalmente com relação a mãe trabalhadora. De acordo com - Estatuto da Criança e do Adolescente (ECA), artigo $9^{\circ}$, o poder público, as instituições e os empregadores propiciaram condições adequadas ao aleitamento materno, inclusive aos filhos de mães submetidas à medida privativa de liberdade. (19)

Com base na promoção de uma alimentação saudável à criança, o MS produz manuais técnicos, publicações e estabelece diretrizes que guiam os 
profissionais de saúde com relação à alimentação e à nutrição de crianças menores de 2 anos que estão em desmame e crianças de 2 a 10 anos. ${ }^{(20)}$

$\mathrm{Na}$ política das Ações Integradas as Doenças Prevalentes na Infância (AIDPI) a proposta é integrar as ações de promoção e prevenção da saúde, com atividades de assistência e tratamento das doenças mais frequentes na infância, reduzindo as mortes e as doenças mais comuns em crianças menores de 5 anos de idade. Essa estratégia fortalece e organiza a atenção primária, capacitando os agentes comunitários de saúde e profissionais da estratégia saúde da família (ESF) para uma atenção de qualidade à criança. ${ }^{(21)}$

O AIDPI objetiva a identificação de sinais que permitam a avaliação e a classificação adequada do quadro clínico, possibilitando uma triagem rápida quanto à natureza da atenção requerida pela criança e o encaminhamento urgente a um hospital, tratamento ambulatorial ou orientação para cuidados e vigilância no domicílio. Além ainda, de fornecer elementos para implantação de uma política de atenção integral à primeira infância no âmbito do SUS e promover, junto a segmentos-chave, como educação, assistente social, justiça, direitos humanos, trabalho e cultura, entre outros, as parcerias necessárias para a implantação de uma atenção a primeira infância. ${ }^{(22,23)}$

O planejamento e o desenvolvimento de ações entre diferentes setores, entre elas a articulação da UBS com os equipamentos sociais, creches, pré -escolas e escolas com vistas à promoção da saúde, são princípios norteadores do cuidado à criança A política nacional de atenção básica prevê, as atividades dos profissionais das equipes de saúde da família, a realização de cuidados de saúde no âmbito da própria unidade de saúde, nos domicílios e nos demais espaços comunitários, escolas, associações, entre outros. ${ }^{(23)}$

Corroborando com este pensamento foi criado o Programa de Saúde na Escola (PSE) que é uma parceria entre o MS e Ministério da Educação. Foi instituído em dezembro de 2007 pelo decreto $n^{\circ} 6286$, e é um programa que considera a escola como um espaço importante e fundamental para as práticas de promoção e prevenção, possibilitando a concretização do desenvolvimento de diversas políticas na saúde infantil, usando a articulação entre a escola e as Unidades Básicas de Saúde (UBS). ${ }^{(24)}$

As causas externas (acidentes e violência) tem destaque na atenção à saúde da criança, pois são responsáveis por gerar problemas sociais, emocionais, psicológicos e cognitivos capazes de causar grande impacto à saúde das pessoas ao longo da sua vida, além de acarretar inúmeras mortes. A faixa etária de o a 9 anos, refere-se à terceira causa de morte e de 10 a 18 anos, constitui-se a primeira causa. ${ }^{(25)}$

Por meio dessas linhas de cuidados o MS reforça a relevância de orientar ações e pactuar estratégias para a redução da morbidade e mortalidade por causas externas. A posição de linha de cuidado para a atenção integral a saúde de crianças, adolescentes e suas famílias em situação de violência organiza-se a partir das dimensões do acolhimento, atendimento, notificações e segmento para a rede de cuidados e proteção social.(11)

A inserção na agenda política brasileira de todas as ferramentas que vislumbram a atenção integral à saúde de crianças, mães e adolescentes é decorrente da mudança de paradigma, expressa na concepção ampliada de saúde como direito social e dever do estado, garantida na constituição de 1988, nas leis orgânicas de saúde e na doutrina da proteção integral ao indivíduo, reconhecidos como sujeitos de direitos à vida, à saúde, à alimentação, à educação, à cultura, ao lazer e a paz.(26)

\section{CONSIDERAÇÕES FINAIS}

A assistência à saúde da criança é de grande importância para que os profissionais fisioterapeutas possam compreender a abordagem da criança em sua totalidade, passando pela dimensão biológica do processo saúde-doença e pelas características assistenciais, através de uma visão retrospectiva da história da atenção à infância. 
A compreensão dessas políticas contribui para a consolidação das ações de saúde e estratégias associadas para a promoção da saúde da população infantil, buscando um melhor entendimento sobre as politicas que englobam a atenção à saúde da criança desde a primeira infância até a politica que abrange o aspecto da adolescência. Desta forma, este trabalho vem mostrar as evidências científicas a cerca da politica do SUS que proporciona um maior conhecimento teórico e prático para nós profissionais de saúde que fazemos parte desse sistema único de saúde.

\section{REFERÊNCIAS}

1. Silva GM, Rasera EF. A construção do SUSproblema no jornal Folha de S. Paulo. Hist. ciênc. saúde-Manguinhos. 2011;21(1):61-76.

2. Mato MC, Lima NT. Fórum. O desafio SUS: $2 O$ anos do Sistema Único de Saúde. Introdução. Cad. saúde pública. 2009;25(7):1611-1613.

3. Brasil. Ministério da Saúde. Saúde da criança: acompanhamento do crescimento e desenvolvimento infantil. Brasília; 2002.

4. Penn H. Primeira infância: a visão do Banco Mundial. Cadernos de Pesquisa. 2002;115.

5. Samico l. et. al. Atenção à saúde da criança: uma análise do grau de implantação e da satisfação de profissionais e usuários em dois municípios do estado de Pernambuco, Brasil. Atenção à saúde da criança. Recife, 2005.

6. Erdmann AL, Sousa FGM. Cuidando da criança na Atenção Básica de Saúde: atitudes dos profissionais da saúde. Mundo saúde. 2009;33(2):150-160.

7. Cervo AL, Bervian PA, Silva R. Metodologia Cientifica. $6^{\mathrm{a}}$ ed. São Paulo: Pearson Prentice; 2007.

8. Moscheta MS, Santos MA. Grupos de apoio para homens com câncer de próstata: revisão integrativa da literatura. São Paulo; 2011.

9. Carvalhal $L M$ et. al. Agenda de compromissos para a saúde integral e redução da mortalidade infantil em um município de Alagoas. Rev. bras. promoç. saúde. 2013; 26(4):530-538.
10. Silva ACMA et. al. Perspectivas de médicos do Programa Saúde da Família acerca das linhas de cuidado propostas pela Agenda de Compromissos para a Saúde Integral da Criança e Redução da Mortalidade Infantil. Cad. saúde pública. 2009; 25(2):349-358.

11. Mendes LC, Santos TT, Bringel FA. Evolução do Programa de Triagem Neonatal no Estado do Tocantins. Arq. bras. endocrinol. metab. 2013;57(2).

12. Cavalcanti PCS et. al. Um modelo lógico da Rede Cegonha. Physis: revista de saude coletiva 2013;23(4):1297-1316.

13. Carneiro RG. Dilemas antropológicos de uma agenda de saúde pública: Programa Rede Cegonha, pessoalidade e pluralidade. Interface comum. saude educ. 2013;17(44):49-59.

14. Goulart LMet. al. Caderneta de Saúde da Criança: avaliação do preenchimento dos dados sobre gravidez, parto e recém- nascido. Rev. paul. pediatr. 2008;26(2):106-12.

15. Alves $C R L$ et. al. Qualidade do preenchimento da Caderneta de Saúde da Criança e fatores associados. Cad. saúde pública. 2009;25(3):583-595.

16. Temporão JG. O Programa Nacional de Imunizações (PNI): origens e desenvolvimento. Hist. ciênc. saúde-Manguinhos. 2003;1O(2):60117.

17. Brasil. Ministério da Saúde. Diretrizes Nacionais para a Atenção Integral à Saúde de Adolescentes e Jovens na Promoção, Proteção e Recuperação da Saúde. Brasília; 2O1Oa.

18. Oliveira MIC, Camacho LAB, Souza IEO. Promoção, proteção e apoio à amamentação na atenção primária à saúde no Estado do Rio de Janeiro, Brasil: uma política de saúde pública baseada em evidência. Cad. saúde pública. 2005;21(6):1901-1910.

19. Almeida GG. et. al. Proteção, promoção e apoio ao aleitamento materno em um hospital universitário. Ciênc. saúde coletiva. 2008;13(2):487-494.

20. Brasil. Ministério da Saúde. Política Nacional de Alimentação e Nutrição. Brasília; 2012.

21. Felisberto E. et. al. Avaliação do processo de implantação da estratégia da Atenção 
Integrada às Doenças Prevalentes da Infância no Programa Saúde da Família, no Estado de Pernambuco, Brasil. Cad. saúde pública. 2002;18(6):1737-1745.

22. PINA JC. et al. Contribuições da estratégia Atenção Integrada às Doenças Prevalentes na Infância ao acolhimento de crianças menores de cinco anos. Acta paul. enferm. 2009; 22(2):142-8.

23. Mendes C. Sumário da Pesquisa Avaliativa da Implantação da Estratégia Brasileirinhas e Brasileirinhos Saudáveis. Rio de Janeiro: Editora Fiocruz; $2 \mathrm{Ol2}$.
24. Brasil. Ministério da Saúde. Linha de Cuidado para a Atenção Integral à Saúde de Crianças, Adolescentes e suas Famílias em Situação de Violências. Brasília; $2010 b$.

25. Santiago LM. Implantação do Programa Saúde na Escola em Fortaleza- CE: atuação de equipe da Estratégia Saúde da Família. Rev. bras. enferm. 2O12;65(6):1O26-9.

26. Raposo C. A Política de Atenção Integral à Saúde do Adolescente e Jovem: uma perspectiva de garantia de direito à saúde? Revista em Pauta. 2009;6(23). 